\title{
The Role of Electron Microscopy in Pediatric Pathology
}

\author{
Eric P. Wartchow
}

Children's Hospital Colorado, Department of Pathology/Laboratory Medicine, Aurora, CO, USA.

Pediatric pathology is a unique specialty within the field of pathology that it is not defined by, or limited to, a specific organ system as with many other pathology specialties (such as pulmonary pathology, neuropathology, hematopathology, etc.). On any given day a pediatric pathologist is likely to encounter disease processes of varying types, including: developmental abnormalities, inherited diseases, acquired diseases, and a wide range of benign and malignant neoplasms. A well-rounded pediatric pathologist will recognize the value of ultrastructural studies and will consider access to an electron microscopy (EM) laboratory an essential requirement for their department. With the modern conveniences of overnight shipping and electronic dissemination of reports the EM lab is no longer required to physically reside within each facility. Whether the ultrastructural evaluation is performed on-site or by a larger reference center, patients will benefit from a close working relationship between the pediatric pathologist and the electron microscopist [1].

Metabolic diseases often present in childhood and can vary greatly in their clinical presentations, pathologic features, and biochemical abnormalities. The recognition of a metabolic disease or identification of a pathologic storage material will incorporate a variety of special techniques including EM. Patients benefit from a fast and accurate diagnosis of metabolic disorders for, if recognized early enough, the natural progression of these diseases can often be modified by medical intervention. In these early stages of metabolic disease the clinical and pathological features may be difficult to recognize and often are overlapping. EM may serve to confirm the presence of a metabolic disease and also help identify the abnormal process. Some of the metabolic processes and diseases, among others, in which EM plays a significant role are: Lysosomal storage diseases (glycogen storage diseases, cholesterol ester storage disease, Krabbe disease, Fabry disease, Gaucher disease, Niemann-Pick disease, mucopolysaccharidoses, neuronal ceroid lipofuscinosis, etc.), Cholestatic liver diseases (Progressive familial intrahepatic cholestasis-Type 1, Alagille syndrome, Alpha-1-antitrypsin deficiency, Zellweger syndromes, etc.), and disorders of metal metabolism (Wilson disease, hemochromatosis, hemosiderosis).

Primary ciliary dyskinesia is an inherited disease of the mucociliary clearance system in which malfunctioning cilia are unable to adequately propel mucous through the airway system. The stagnant mucous collects pathogens which accumulate within the airways triggering the infection/inflammation cycle and which eventually leads to scarring. Identification of the abnormalities within individual cilia requires the use of the electron microscope. The ciliary axoneme comprises microtubules arranged in the common 9+2 arrangement. The nine microtubule doublets surround two single microtubules and are held in place by various structural proteins. Cilia movement is conferred by groups of dynein proteins which appear as "arms" extending from each outer microtubule doublet toward the adjacent doublet. Genetic mutations in genes encoding any of these structural or movement-associated proteins inhibit ciliary motion and results in primary ciliary dyskinesia. The role of the electron microscopist is to identify any specific abnormality which may represent the morphologic expression of a mutation and be the causative agent of the disease. 
Discrimination of childhood tumors is one of the more challenging diagnostic concerns facing a pediatric pathologist. Small round cell tumors of childhood, because of their primitive or undifferentiated appearance when viewed by light microscopy, can be particularly difficult. They are generally highly malignant and occur in pediatric populations. The most common tumor types within the category are: Ewing sarcoma/PNET, neuroblastoma, rhabdomyosarcoma, Wilms' tumor, synovial sarcoma and desmoplastic small round cell tumor. The integration of routine histologic examination with ultrastructural, cytogenetic, immunocytologic and molecular genetic studies is required to confidently offer an accurate diagnosis. EM is particularly useful in instances when tumor cells are extremely undifferentiated and unreactive to immuno labeling, when the combination of other techniques produces equivocal results, or when only a limited quantity of tissue is available for triage.

Routine histologic examination of tissue by a pathologist will oftentimes result in a definitive diagnosis; however, with the increasing complexity of our understanding of human health and the necessity for narrower classifications and subtyping of various disease processes the pathologist is best prepared to face diagnostic dilemmas when they have a clear understanding of the many tools available to them and the knowledge to select the best approach for specific cases [2]. In past years, EM enjoyed notoriety as perhaps the single most important ancillary diagnostic technique. As others, such as immunohistochemistry, fluorescence in-situ hybridization, flow cytometry and molecular genetics were developed each was labeled "the next big thing" that would replace previously favored techniques. These predictions were proven to be impractical and ill-conceived. EM continues to be employed to provide definitive diagnoses or to guide the responsible usage of other diagnostic techniques for many disease processes and for this reason EM will long remain an essential tool in the diagnostic arsenal of the practicing pediatric pathologist.

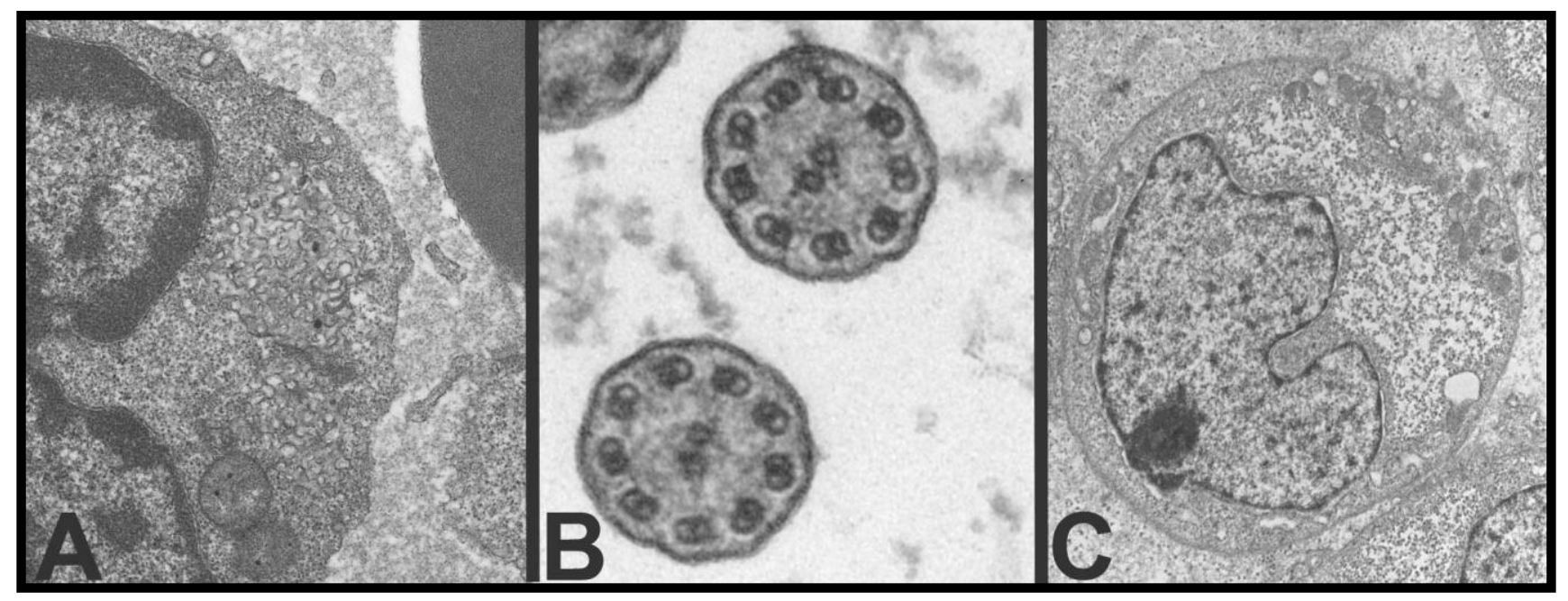

Figure 1. Examples of electron microscopy in pediatric pathology. A: Neuronal ceroid lipofuscinosis; CLN2. B: Primary ciliary dyskinesia. C: Ewing sarcoma.

\section{References:}

[1] G. Mierau, E. Wartchow, J. Hicks in "Pediatric Pathology $4^{\text {th }}$ Edition", ed A. Husain, JT Stocker, L Dehner, (Wolters Kluwer, Philadelphia) p. 51-59.

[2] J. Hicks. Ultrastructural Pathology 27 (2003), p. 287-288. 